(n)

trobertivier Journal of Nonlinear Mathematical Physics

\title{
Finite and infinite systems of nonlinearly-coupled ordinary differential equations, the solutions of which feature remarkable Diophantine findlings
}

Francesco Calogero

To cite this article: Francesco Calogero (2018) Finite and infinite systems of nonlinearlycoupled ordinary differential equations, the solutions of which feature remarkable Diophantine findings, Journal of Nonlinear Mathematical Physics 25:3, 433-441, DOI: https://doi.org/10.1080/14029251.2018.1494753

To link to this article: https://doi.org/10.1080/14029251.2018.1494753

Published online: 04 January 2021 


\title{
Finite and infinite systems of nonlinearly-coupled ordinary differential equations, the solutions of which feature remarkable Diophantine findings
}

\author{
Francesco Calogero \\ Dipartimento di Fisica, Università di Roma "La Sapienza" \\ Istituto Nazionale di Fisica Nucleare, Sezione di Roma \\ francesco.calogero@romal.infn.it,francesco.calogero@uniromal.it
}

Received 19 January 2018

Accepted 16 March 2018

\begin{abstract}
We use previous results concerning the time evolution of the zeros $x_{n}(t)$ of time-dependent polynomials $p_{N}(z ; t)$ or entire functions $F(z ; t)$ of the complex variable $z$, in order to identify lots of nonlinearly-coupled, finite or infinite, systems of Ordinary Differential Equations the solutions of which feature remarkable Diophantine properties.
\end{abstract}

\section{Introduction, and some representative results}

Certain useful differential identities associated with the time evolution of the $N$ zeros $x_{n}(t)$ of time dependent polynomials $p_{N}(z ; t)$ of degree $N$ in the complex variable $z$ were recently identified and utilized to obtain nonlinear systems of $N$ nonlinearly-coupled Ordinary Differential Equations (ODEs) satisfied by these $N$ zeros $x_{n}(t)$ and featuring interesting properties (for instance isochronous evolutions) [1]. In the present paper we show how to use these differential identities in order to identify systems of $N$ nonlinearly-coupled ODEs—satisfied by these zeros $x_{n}(t)$ 一the solutions of which feature remarkable Diophantine properties.

Analogous findings are moreover obtained for the systems of infinitely many nonlinearly coupled ODEs satisfied by the infinitely many zeros $x_{n}(t)$ of time-dependent entire functions $F(z ; t)$ of the complex variable $z$, by utilizing certain recently identified differential identities characterizing the time evolution of these zeros [2].

Notation 1.1 Throughout this paper $t$ is a real variable which ranges from 0 to 1 (it is convenient to consider it a "dimensionless time"); and the notation $\left\{\left\{z_{n}\right\}\right\}$ indicates the unordered set of the quantities $z_{n}$ labeled by the index $n$ (which may take a finite, or an infinite, set of integer values; see below).

Typical examples of findings of the first kind are detailed by the following 2 Propositions, where $N$ is an arbitrary positive integer $(N>1)$ and the indices $n$ and $\ell$ run over the integers from 1 to $N$ (unless otherwise indicated).

Proposition 1.1. Consider the system of $N$ nonlinearly coupled first-order $O D E s$

$$
\dot{x}_{n}(t)=\frac{\prod_{\ell=1}^{N}\left[x_{n}(t)-x_{\ell}(0)\right]-\prod_{\ell=1}^{N}\left[x_{n}(t)-\ell\right]}{\prod_{\ell=1, \ell \neq n}^{N}\left[x_{n}(t)-x_{\ell}(t)\right]}
$$


where of course (here and hereafter) a superimposed dot denotes (partial) differentiation with respect to the real independent variable $t$, and the $N$ initial (possibly complex) values $x_{n}(0)$ of the dependent variables $x_{n}(t)$ are assigned arbitrarily (but all different among themselves$x_{n}(0) \neq x_{\ell}(0)$ if $n \neq \ell$-to avoid that the denominator in the right-hand side of (1.1a) vanish at the initial time $t=0)$.

Then the values $x_{n}(1)$ of the dependent variables $x_{n}(t)$ at $t=1$ feature the remarkable Diophantine property to coincide with the first $N$ integers,

$$
\left\{\left\{x_{n}(1)\right\}\right\}=\{\{n\}\} .
$$

Remark 1.1. For $N=2$ the initial-values problem for the system (1.1a) can be explicitly solved:

$$
\begin{array}{r}
x_{1,2}(t)=\frac{-b(t) \pm\left\{[b(t)]^{2}-4 c(t)\right\}^{1 / 2}}{2}, \\
b(t)=(t-1)\left[x_{1}(0)+x_{2}(0)\right]-3 t, \\
c(t)=(1-t) x_{1}(0) x_{2}(0)+2 t ;
\end{array}
$$

and the diligent reader will readily verify that at $t=1$ it yields the outcome (1.1b) (with $n=1,2$ ).

Proposition 1.2. Consider the system of $N$ nonlinearly-coupled second-order $O D E s$

$$
\ddot{x}_{n}(t)=\sum_{\ell=1, \ell \neq n}^{N}\left[\frac{2 \dot{x}_{n}(t) \dot{x}_{\ell}(t)}{x_{n}(t)-x_{\ell}(t)}\right]
$$

with the following assignment of the "initial velocities"

$$
\dot{x}_{n}(0)=\frac{-\prod_{\ell=1}^{N}\left[x_{n}(0)-\ell\right]}{\prod_{\ell=1, \ell \neq n}^{N}\left[x_{n}(0)-x_{\ell}(0)\right]},
$$

and the $N$ initial (possibly complex) values $x_{n}(0)$ of the dependent variables $x_{n}(t)$ assigned arbitrarily (but all different among themselves, to avoid that the denominator in the right-hand side of (1.3b) vanish).

Then, as in the case of Proposition 1.1, the values $x_{n}(1)$ of the dependent variables $x_{n}(t)$ at $t=1$ feature the remarkable Diophantine property to coincide with the first $N$ integers, see (1.1b).

Remark 1.2. For $N=2$ the initial-values problem for the system (1.3) can be explicitly solved:

$$
\begin{aligned}
x_{1,2}(t) & =\frac{-\beta(t) \pm\left\{[\beta(t)]^{2}-4 \gamma(t)\right\}^{1 / 2}}{2}, \\
\beta(t) & =-\left[x_{1}(0)+x_{2}(0)\right]-\left[\dot{x}_{1}(0)+\dot{x}_{2}(0)\right] t, \\
\gamma(t) & =x_{1}(0) x_{2}(0)+\left[\dot{x}_{1}(0) x_{2}(0)+x_{1}(0) \dot{x}_{2}(0)\right] t, \\
\dot{x}_{n}(0) & =\frac{-\left[x_{n}(0)\right]^{2}+3 x_{n}(0)-2}{x_{n}(0)-x_{n+1}(0)}, n=1,2 \bmod [2] ;
\end{aligned}
$$


and the reader will again readily verify that at $t=1(1.4 \mathrm{a})$ yields the outcome $(1.1 \mathrm{~b})$ (with $n=1,2)$.

Typical examples of findings of the second kind are detailed by the following 2 Propositions, where the indices $n$ and $\ell$ run now over all integers from $-\infty$ to $+\infty$ (unless otherwise indicated).

Proposition 1.3. Consider the system of infinitely many nonlinearly-coupled first-order ODEs:

$$
\dot{x}_{n}(t)=\frac{x_{n}(t)\left(\cos \left[\pi x_{n}(t)\right]-\prod_{\ell=-\infty}^{\ell=+\infty}\left\{1-\left[x_{n}(t) / x_{\ell}(0)\right]\right\}\right)}{\prod_{\ell=-\infty, \ell \neq n}^{\ell=+\infty}\left\{1-\left[x_{n}(t) / x_{\ell}(t)\right]\right\}},
$$

where the initial (possibly complex) values $x_{n}(0)$ of the infinitely many dependent variables $x_{n}(t)$ are assigned arbitrarily (but all different among themselves, and moreover so that the product in the numerator in the right-hand side of (1.5a) converge absolutely).

Then the infinitely many values $x_{n}(1)$ of the dependent variables $x_{n}(t)$ at $t=1$ feature the remarkable Diophantine property to coincide with the infinitely many zeros of the function $\cos (\pi x)$, namely

$$
\left\{\left\{x_{n}(1)\right\}\right\}=\left\{\left\{\frac{2 n+1}{2}\right\}\right\}
$$

Proposition 1.4. Consider the system of infinitely many nonlinearly coupled second-order ODEs

$$
\ddot{x}_{n}(t)=\frac{2\left[\dot{x}_{n}(t)\right]^{2}}{x_{n}(t)}+\sum_{\ell=-\infty, \ell \neq n}^{+\infty}\left[\frac{2 x_{n}(t) \dot{x}_{n}(t) \dot{x}_{\ell}(t)}{x_{\ell}(t)\left[x_{n}(t)-x_{\ell}(t)\right]}\right]
$$

with the initial velocities

$$
\dot{x}_{n}(0)=\frac{x_{n}(0) \cos \left[\pi x_{n}(0)\right]}{\prod_{\ell=-\infty, \ell \neq n}^{\ell=+\infty}\left\{1-\left[x_{n}(0) / x_{\ell}(0)\right]\right\}},
$$

and with the infinitely many initial data $x_{n}(0)$ assigned arbitrarily (but all different among themselves, and moreover so that the product in the denominator in the right-hand side of (1.6b) converge absolutely).

Then the infinitely many values $x_{n}(1)$ of the dependent variables $x_{n}(t)$ at $t=1$ feature again (as in Proposition 1.3) the remarkable Diophantine property to coincide with the infinitely many zeros of the function $\cos (\pi x)$, see (1.5b).

Remark 1.3. Let us emphasize that these 4 Propositions hold for any arbitrary assignment of the initial data $x_{n}(0)$; this fact seems a priori less than obvious, although of course, as all correct mathematical findings, it shall be a posteriori obvious (after it has been proven, see below).

These results are in fact quite special instances of more generals findings, as explained in the following Section 2 ("More general results and their proofs"). Section 3 ("Outlook") contains some final considerations. 


\section{More general results and their proofs}

In this Section 2 more general results are reported and proven, which obviously also imply the findings reported in Section 1, as well as many analogous results that the interested reader will easily formulate. These findings are based on the time evolution of the $N$ zeros of a time dependent polynomial $p_{N}(z ; t)$ of degree $N$ in the complex variable $z$ (with $N$ an arbitrary integer, $N>1$ ), respectively on the time evolution of the infinitely many zeros of the time dependent function $F(z ; t)$ which is entire in its dependence upon the complex variable $z$; with both $p_{N}(z ; t)$ and $F(z ; t)$ featuring an extremely simple time dependence. These two cases are treated separately, in Subsection 2.1 respectively in Subsection 2.2.

\subsection{The time evolution of the $N$ zeros of the polynomial $p_{N}(z ; t)$}

The treatment in this Subsection 2.1 is largely based on previous findings, see below, hence it is rather terse.

The starting point in this Subsection 2.1 is the polynomial

$$
p_{N}(z ; t)=\prod_{n=1}^{N}\left[z-x_{n}(t)\right],
$$

which we write here in terms of its $N$ zeros $x_{n}(t)$. In this Subsection 2.1 indices such as $n, \ell$ run over the integers from 1 to $N$ unless otherwise indicated. Note that this definition of the polynomial $p_{N}(z ; t)$ implies that this polynomial is monic, namely that the coefficient of its highest power $z^{N}$ is unity.

It is by now well known (see [1] and references therein) that there hold then the following two identities which relate as follows the time evolution of the $N$ zeros $x_{n}(t)$ to the overall time evolution of the first and second partial time derivatives of the polynomial $p_{N}(z ; t)$ evaluated at $z=x_{n}(t)$ :

$$
\begin{gathered}
\dot{x}_{n}(t)=-\frac{p_{N, t}\left[x_{n}(t) ; t\right]}{\prod_{\ell=1, \ell \neq n}^{N}\left[x_{n}(t)-x_{\ell}(t)\right]}, \\
\ddot{x}_{n}(t)=\sum_{\ell=1, \ell \neq n}^{N}\left[\frac{2 \dot{x}_{n}(t) \dot{x}_{\ell}(t)}{x_{n}(t)-x_{\ell}(t)}\right]-\frac{p_{N, t t}\left[x_{n}(t) ; t\right]}{\prod_{\ell=1, \ell \neq n}^{N}\left[x_{n}(t)-x_{\ell}(t)\right]} .
\end{gathered}
$$

Of course the notations $p_{N, t}\left[x_{n}(t) ; t\right] \equiv \partial p_{N}(z ; t) /\left.\partial t\right|_{z=x_{n}(t)}$ respectively $p_{N, t t}\left[x_{n}(t) ; t\right] \equiv$ $\partial^{2} p_{N}(z ; t) /\left.\partial t^{2}\right|_{z=x_{n}(t)}$ denote the first respectively the second partial time derivatives of the polynomial $p_{N}(z ; t)$ evaluated at $z=x_{n}(t)$.

We now formulate the following

Lemma 2.1. Consider the following two systems of $N$ nonlinearly coupled ODEs:

(i) the system of $N$ first-order $O D E s$

$$
\dot{x}_{n}(t)=\frac{\varphi_{N}\left[x_{n}(t)\right]-\Phi_{N}\left[x_{n}(t)\right]}{\prod_{\ell=1, \ell \neq n}^{N}\left[x_{n}(t)-x_{\ell}(t)\right]} ;
$$


(ii) the system of $N$ second-order $O D E s$

$$
\ddot{x}_{n}(t)=\sum_{\ell=1, \ell \neq n}^{N}\left[\frac{2 \dot{x}_{n}(t) \dot{x}_{\ell}(t)}{x_{n}(t)-x_{\ell}(t)}\right]
$$

complemented by the following assignment of the "initial velocities"

$$
\dot{x}_{n}(0)=\frac{-\Phi_{N}\left[x_{n}(0)\right]}{\prod_{\ell=1, \ell \neq n}^{N}\left[x_{n}(0)-x_{\ell}(0)\right]} .
$$

In both cases (i) and (ii) the two monic polynomials $\varphi_{N}(z)$ and $\Phi_{N}(z)$ are defined as follows:

$$
\begin{aligned}
\varphi_{N}(z) & =\prod_{\ell=1}^{N}\left[z-x_{\ell}(0)\right], & \varphi_{N}\left[x_{n}(0)\right] & =0, \\
\Phi_{N}(z) & =\prod_{\ell=1}^{N}\left(z-\xi_{\ell}\right), & \Phi_{N}\left(\xi_{n}\right) & =0 .
\end{aligned}
$$

In all these formulas the $N$ "initial data" $x_{n}(0)$ can be arbitrarily assigned, except for the restriction that they be all different among themselves (to exclude that the denominators in the right-hand sides of the equations of motion (2.4) and (2.5) vanish at $t=0$ ); while the $N$ zeros $\xi_{n}$ can as well be arbitrarily assigned, so that $\Phi_{N}(z)$ is the arbitrary polynomial of degree $N$ in $z$ characterized by these $N$ zeros, see (2.6b).

Then the unordered set of the $N$ values attained by the solutions of either one of these two systems of $N$ nonlinearly coupled ODEs at $t=1$ coincide with the unordered set of the $N$ zeros $\xi_{n}$ of the polynomial $\Phi_{N}(z)$ :

$$
\left\{\left\{x_{n}(1)\right\}\right\}=\left\{\left\{\xi_{n}\right\}\right\}
$$

Let us re-emphasize that, in all these formulas, the "initial values" $x_{n}(0)$ are $N$ (possibly complex) numbers which can be arbitrarily assigned - except for the requirement that they be all different among themselves, $x_{n}(0) \neq x_{\ell}(0)$ if $n \neq \ell$.

To prove this Lemma 2.1 we make the following assignment for the a priori arbitrary monic polynomial $p_{N}(z ; t)$ :

$$
p_{N}(z ; t)=(1-t) \varphi_{N}(z)+t \Phi_{N}(z) .
$$

Note that this relation (2.8) is consistent with the monic character of $p_{N}(z ; t)$, see (2.1), as implied by the monic character of the two polynomials $\varphi_{N}(z)$ and $\Phi_{N}(z)$, see (2.6).

It is plain that this assignment (2.8) implies the following 4 relations:

$$
\begin{aligned}
p_{N, t}(z ; t) & =\Phi_{N}(z)-\varphi_{N}(z), & p_{N, t t}(z ; t) & =0, \\
p_{N}(z ; 0) & =\varphi_{N}(z), & p_{N}(z ; 1) & =\Phi_{N}(z) .
\end{aligned}
$$

We now note that the insertion of the first of these 4 formulas (2.9) in the identity (2.2) yields the system of $N$ nonlinearly-coupled first-order ODEs (2.4); the insertion of the second of these 4 formulas (2.9) in the identity (2.3) yields the system of $N$ nonlinearly-coupled second-order ODEs 
(2.5a), which must of course be complemented by the assignment (2.5b) of the $N$ "initial velocities" $\dot{x}_{n}(0)$ as implied—at $t=0$-by (2.4) together with (2.6a), the consistency of this assignment (2.6a) being demonstrated by the third of these 4 formulas (2.9) with (2.1) at $t=0$; and finally the fourth of these 4 formulas (2.9) implies (via (2.1) and (2.6b)) the relation (2.7), proving Lemma 2.1.

We are now ready to prove Proposition 1.1 respectively Proposition 1.2. Indeed their validity is immediately implied by this Lemma 2.1 — see (2.7) and (2.4) respectively (2.5)—via the assignments (2.6a) and (2.6b) with $\xi_{n}=n$.

And of course the imaginative reader can formulate other analogous, but more general, findings than those detailed in Propositions 1.1 and 1.2, by making other assignments $\xi_{\ell}=f_{\ell}(\ell)$ of the, $a$ priori arbitrary, $N$ numbers $\xi_{\ell}$; for instance each function $f_{\ell}(\lambda)$ could be an arbitrary polynomial with integer coefficients in its argument $\lambda$.

We complete this Subsection 2.1 with the following remark.

Remark 2.1. If over the time evolution it happens that at some time $\tilde{t}$ (in the relevant range $0<$ $\tilde{t} \leq 1)$ different zeros collide-say, $x_{\tilde{n}}(\tilde{t})=x_{\tilde{\ell}}(\tilde{t})$ with $\tilde{n} \neq \tilde{\ell}$-the systems of differential equations (1.1a) and (1.3a) clearly run at $t=\tilde{t}$ into a singularity, due to a blow-up of the right-hand sides of the two ODEs with $n=\tilde{n}$ and $\ell=\tilde{\ell}$. But this fact does not entail a blow-up at $t=\tilde{t}$ of the corresponding solutions $x_{\tilde{n}}(t)$ and $x_{\tilde{\ell}}(t)$, which indeed are, for all time, the zeros of a polynomial of degree $N$ with finite coefficients, see (2.1), (2.8) and (2.6). What generally instead happens is that the functions $x_{\tilde{n}}(t)$ and $x_{\tilde{\ell}}(t)$ feature a branch point at $t=\tilde{t}$, and that for $t>\tilde{t}$ the assignment of the labels $\tilde{n}$ and $\tilde{\ell}$ to these two solutions can be re-assigned arbitrarily. This does not have much relevance for the findings reported above, because the set of the $N$ zeros $x_{n}$ of a polynomial $p_{N}(z)$, see (2.1), are by definition an unordered set $\left\{\left\{x_{n}\right\}\right\}$.

\subsection{The time evolution of the infinitely many zeros of the entire function $F(z ; t)$}

The treatment in this Subsection 2.2 is again largely based on previous findings [2]; hence it is again rather terse.

The starting point is the following standard representation of the function $F(z ; t)$, which is, for all values of the real variable $t$ in the interval $0 \leq t \leq 1$, an a priori arbitrary but entire function of the complex variable $z$, admitting moreover the following representation in terms of its (generally infinitely many, generally complex) zeros $x_{\ell}(t)$ :

$$
F(z ; t)=\prod_{\ell=-\infty}^{+\infty}\left[1-\frac{z}{x_{\ell}(t)}\right]
$$

so that

$$
\begin{gathered}
F\left[x_{n}(t) ; t\right]=0, \\
F(0 ; t)=F(0 ; 0)=1 .
\end{gathered}
$$

Of course throughout this Subsection 2.2 the indices $n$ and $\ell$ take all integer values in the range from $-\infty$ to $+\infty$ (unless otherwise indicated).

Note that here and hereafter we make for simplicity the additional assumption that $F(z ; t)$ does not feature a vanishing zero, $F(0 ; t) \neq 0$; and that the above assignment entails an additional simplification (see $(2.10 \mathrm{c})$ ), entailing no significant restriction for our treatment. Note that the assumption 
that $F(z ; t)$ is an entire function of $z$ implies the absolute convergence of the product in the righthand side of (2.10a) for all (complex) values of $z$.

There hold then-see the two equations (4a) respectively (4b) of [2] - the following two identities which relate as follows the time evolution of the infinitely many zeros $x_{n}(t)$ to the overall time evolution of the first and second partial $t$-derivatives of the function $F(z ; t)$ evaluated at $z=x_{n}(t)$ :

$$
\begin{gathered}
\dot{x}_{n}(t)=x_{n}(t) F_{t}\left[x_{n}(t) ; t\right]\left\{\prod_{\ell=-\infty, \ell \neq n}^{+\infty}\left[1-\frac{x_{n}(t)}{x_{\ell}(t)}\right]\right\}^{-1} ; \\
\ddot{x}_{n}(t)=\frac{2\left[\dot{x}_{n}(t)\right]^{2}}{x_{n}(t)}+\sum_{\ell=-\infty, \ell \neq n}^{+\infty}\left\{\frac{2 x_{n}(t) \dot{x}_{n}(t) \dot{x}_{\ell}(t)}{x_{\ell}(t)\left[x_{n}(t)-x_{\ell}(t)\right]}\right\} \\
+x_{n}(t) F_{t t}\left[x_{n}(t) ; t\right]\left\{\prod_{\ell=-\infty, \ell \neq n}^{+\infty}\left[1-\frac{x_{n}(t)}{x_{\ell}(t)}\right]\right\}^{-1} .
\end{gathered}
$$

Here and hereafter of course $F_{t}\left[x_{n}(t) ; t\right]$, respectively $F_{t t}\left[x_{n}(t) ; t\right]$, are the first, respectively the second, partial $t$-derivatives of the function $F(z ; t)$ evaluated at $z=x_{n}(t)$.

We now formulate the following:

Lemma 2.2. Consider the following two systems of $N$ nonlinearly coupled ODEs:

(i) the system of infinitely many first-order ODEs

$$
\dot{x}_{n}(t)=x_{n}(t)\left\{\Phi\left[x_{n}(t)\right]-\varphi\left[x_{n}(t)\right]\right\}\left\{\prod_{\ell=-\infty, \ell \neq n}^{+\infty}\left[1-\frac{x_{n}(t)}{x_{\ell}(t)}\right]\right\}^{-1} ;
$$

(ii) the system of infinitely many second-order ODEs

$$
\ddot{x}_{n}(t)=\frac{2\left[\dot{x}_{n}(t)\right]^{2}}{x_{n}(t)}+\sum_{\ell=-\infty, \ell \neq n}^{+\infty}\left\{\frac{2 x_{n}(t) \dot{x}_{n}(t) \dot{x}_{\ell}(t)}{x_{\ell}(t)\left[x_{n}(t)-x_{\ell}(t)\right]}\right\}
$$

complemented by the following assignment of the "initial velocities"

$$
\dot{x}_{n}(0)=x_{n}(0) \Phi\left[x_{n}(0)\right]\left\{\prod_{\ell=-\infty, \ell \neq n}^{+\infty}\left[1-\frac{x_{n}(0)}{x_{\ell}(0)}\right]\right\}^{-1} .
$$

In both cases (i) and (ii), the two entire function $\varphi(z)$ and $\Phi(z)$ are defined as follows:

$$
\begin{gathered}
\varphi(z)=\prod_{\ell=-\infty}^{+\infty}\left[1-\frac{z}{x_{\ell}(0)}\right], \quad \varphi(0)=1, \quad \varphi\left[x_{n}(0)\right]=0, \\
\Phi(z)=\prod_{\ell=-\infty}^{+\infty}\left[1-\frac{z}{\xi_{\ell}}\right], \quad \Phi(0)=1, \quad \Phi\left(\xi_{n}\right)=0 .
\end{gathered}
$$

Note that we are assuming that the entire function $\varphi(z)$ is defined in terms of the infinitely many initial values $x_{n}(0)$ of the dependent variables $x_{n}(t)$, which are its zeros (see (2.13a)) and which can be assigned arbitrarily, but of course so as to guarantee the absolute convergence of the product in the definition of $\varphi(z)$, see (2.13a); and note moreover that these zeros $x_{n}(0)$ must be all different among themselves (i.e., $x_{n}(0) \neq x_{\ell}(0)$ if $n \neq \ell$ ), to avoid the blowing up of the right-hand side of 
(2.12b). While the other function $\Phi(z)$ is an arbitrary entire function (up to its normalization at the origin, see (2.13b)) characterized by the a priori arbitrary set of its zeros $\xi_{n}-$ which must of course also be assigned so as to guarantee the absolute convergence of the infinite product in the definition of $\Phi(z)$, see (2.13b).

Then the unordered set of the infinitely many values attained by the solutions of either one of these two systems of $N$ nonlinearly coupled ODEs at $t=1$ coincide with the unordered set of the infinitely many numbers $\xi_{n}$ :

$$
\left\{\left\{x_{n}(1)\right\}\right\}=\left\{\left\{\xi_{n}\right\}\right\}
$$

To prove this Lemma 2.2 we make the following assignment (analogous to (2.8)) for the a priori arbitrary entire function $F(z ; t)$ :

$$
F(z ; t)=(1-t) \varphi(z)+t \Phi(z),
$$

where the two ( $t$-independent) functions $\varphi(z)$ and $\Phi(z)$ are both entire, being characterized by the set of their zeros as indicated above, see (2.13).

This ansatz of course implies via (2.10d) and (2.10e) the validity of (2.11), and (2.12a)-(2.12b) and (consistently with (2.13a))

$$
F(z ; 0)=\varphi(z)=\prod_{\ell=-\infty}^{+\infty}\left[1-\frac{z}{x_{\ell}(0)}\right]
$$

hence

$$
F\left[x_{n}(0) ; 0\right]=\varphi\left[x_{n}(0)\right]=0 ;
$$

and

$$
F(z ; 1)=\Phi(z)=\prod_{\ell=-\infty}^{+\infty}\left(1-\frac{z}{\xi_{\ell}}\right)
$$

hence (compare (2.10a) with (2.16c)), the validity of the relation (2.14), thereby proving Lemma 2.2.

Let us re-emphasize that this conclusion holds for any arbitrary assignment of the initial data $x_{n}(0)$, provided it is consistent with the absolute convergence of the product in the right-hand side of the first relation (2.13a) and with the requirement that all these infinitely many values $x_{n}(0)$ be all different among themselves (so that the right-hand side of (2.12b) does not blow up).

The proofs of both Proposition 1.3 and Proposition 1.4 are then immediately implied by Lemma 2.2, via the assignment

$$
\Phi(x)=\cos (\pi x)
$$

implying of course (see (2.13b)) that the numbers $\xi_{n}$ are the zeros of $\cos (\pi x)$, see (1.5b).

We complete this Section 2 with the following remark.

Remark 2.2. The interested reader will have no difficulty to extend Remark 2.1 from the context of Subsection 2.1 to the context of this Subsection 2.2. 


\section{Outlook}

A potential application of the findings reported above is the possibility to use them to test the accuracy of standard computer programs to integrate numerically systems of nonlinearly coupled ODEs. In this connection it might be somewhat relevant to note that the polynomial $\Phi_{N}(z)$ (see (2.13b)) with $N=20$ and $\xi_{n}=n$ happens to be just the "perfidious" Wilkinson polynomial [3].

A second development that the interested reader might pursue is the variant of the above approach-we refer for simplicity to the treatment of Subsection 2.1-that obtains if the (monic) polynomial $p_{N}(z, t)$ linear in $t$, see (2.8), is replaced by a (monic) polynomial simply periodic in $t$, say

$$
p_{N}(z ; t)=[1-\sin (\omega t)] \varphi_{N}(z)+\sin (\omega t) \Phi_{N}(z)
$$

\section{Acknowledgements}

It is a pleasure to thank my former student Matteo Sommacal who some time ago introduced me to the "perfidious" properties of the Wilkinson polynomial in the context of numerical analysis. And also thanks to two anonymous Referees who helped me to improve my presentation.

\section{References}

[1] F. Calogero, "New solvable variants of the goldfish many-body problem", Studies Appl. Math. 137 (1), 123-139 (2016), DOI:10.1111/sapm.12096; Zeros of polynomials and solvable nonlinear evolution equations, 170-page book, Cambridge University Press (in press) (2018).

[2] F. Calogero, "Zeros of entire functions and related systems of infinitely many nonlinearly coupled evolution equations", Theor. Math. Phys. (in press) (2018).

[3] J.H. Wilkinson, "The perfidious polynomial", in Studies in Numerical Analysis, vol. 24, pp. 1-28, 1984; G.H. Golub (editor), Mathematical Association of America, Washington DC, USA. 